\title{
Aquaporin expression in breast cancer and their involvement in bleb formation, cell motility and invasion in endocrine resistant variant cells
}

\author{
AYAH E. AHMAD ${ }^{1}$, MAITHAM A. KHAJAH ${ }^{2}$, SARAH KHUSHAISH ${ }^{2}$ and YUNUS A. LUQMANI ${ }^{1}$ \\ Departments of ${ }^{1}$ Pharmaceutical Chemistry and ${ }^{2}$ Pharmacology and Therapeutics, \\ Faculty of Pharmacy, Kuwait University, Safat 13110, Kuwait
}

Received September 3, 2019; Accepted January 16, 2020

DOI: $10.3892 /$ ijo.2020.4976

\begin{abstract}
Estrogen receptor (ER)-silenced breast cancer cell lines exhibit endocrine resistance and morphological changes from an epithelial to a mesenchymal phenotype. These cells also display increased motility and invasive properties that are further accentuated by exposure to an alkaline $\mathrm{pH}$, exhibiting dynamic plasma membrane blebbing and cytoplasmic streaming. These latter morphological changes are hypothesized to involve substantial water movement across the plasma membrane, contributing to bleb formation; this may involve aquaporin channel proteins (AQPs). AQP 1, 3, 4 and 5 expression/localization was examined via reverse transcription-quantitative PCR, western blotting and confocal microscopy in endocrine-sensitive (YS1.2) and -resistant (pII and MDA-MB-231) breast cancer cells, as well as normal breast epithelial cells (MCF10A). The effects of osmotic changes on bleb formation were examined via live cell imaging. AQP3 protein expression was knocked down by small interfering RNA (siRNA) transfection, and the effect of its reduced expression on bleb formation, cell motility and invasion were determined via immunofluorescence, scratch and Cultrex assays, respectively. Expression of the four AQPs varied across the different cell lines, and exhibited nuclear, cytoplasmic and membranous localization. Osmotic changes affected the formation of blebs. In pII cells exposed to alkaline $\mathrm{pH}, \mathrm{AQP} 3$ was observed to be redistributed from the nucleus into the newly formed blebs. siRNA-mediated knockdown of AQP3 in pII cells significantly reduced cellular blebbing induced by alkaline $\mathrm{pH}$, as well as motility and invasion. These data suggested that AQP3, and potentially other aquaporins, may participate in the processes leading to blebbing of
\end{abstract}

Correspondence to: Professor Yunus A. Luqmani, Department of Pharmaceutical Chemistry, Faculty of Pharmacy, Kuwait University, Safat 13110, Kuwait

E-mail: yunus@hsc.edu.kw

Key words: breast cancer, aquaporin, motility, bleb formation, $\mathrm{pH}$, invasion, endocrine resistance endocrine-resistant cells which is proposed to be a mechanism that drives tumor metastasis.

\section{Introduction}

Breast cancer is the most commonly diagnosed neoplasm and the leading cause of cancer mortality among females worldwide $(1,2)$. Clinically, it is divided into three main subtypes based on hormone receptor expression (3-6). One of the main characteristics of aggressive breast cancers is epithelial-mesenchymal transition (EMT), via which cells transition from an epithelial to a mesenchymal phenotype, a phenomenon that has been closely linked to metastasis, drug resistance and cancer recurrence, and reported to result in poor prognosis (7). Hallmarks of EMT include the downregulation of E-cadherin, decreased expression of claudins and occludins at tight junctions, and repression of genes encoding desmoplakin and plakophilin desmosomes (8). Reductions in the levels of these epithelial proteins disrupt cell junction complexes and cytoskeletal connections (9); occurring in parallel with activation of genes such as $\mathrm{N}$-cadherin, vimentin, fibronectin, $\alpha$-smooth muscle actin and matrix metalloproteinases (MMPs), this gives cells a mesenchymal-like phenotype $(10,11)$. EMT increases cancer cell motility and facilitates invasive behavior by enabling cells to penetrate through the extracellular matrix, and invade lymphatic and vascular elements (7).

In the process of studying therapeutic resistance to endocrine therapy, several cell lines have been established in our laboratory via short hairpin RNA (shRNA)-mediated knockdown of the estrogen receptor (ER) in parental endocrine-sensitive MCF-7 breast cancer cells, resulting in cells resistant to both tamoxifen and estrogen, and associated with EMT, leading to increased motility and invasion $(8,11,12)$. One of the main characteristics of these endocrine-resistant cells, but not of endocrine-sensitive breast cancer cells or normal breast epithelial cells, is the induction of a remarkable alteration in their morphology, including cell rounding and the formation of dynamic actin-rich blebs along the outer membrane upon brief exposure to alkaline but not acidic $\mathrm{pH}$ conditions $(13,14)$. Under such conditions, ER-silenced pII cells exhibit enhanced motility and migration, in part through increased MMP activity (12). These blebs can be observed at the leading 
edge of cells moving towards a chemoattractant source in an amoeboid-like manner (13-15). Bleb formation can be reversed or prevented by inhibitors of cytoplasmic streaming or drugs known to inhibit certain ion channels; the most effective of these is ouabain, a potent inhibitor of $\mathrm{Na}^{+} / \mathrm{K}^{+}$ATPase $(13,14)$. The reversibility of bleb formation - which disappear upon returning the cells to $\mathrm{pH} 7.4$ - suggests that this may be a physiological process to promote cellular motility in order to enable cells to move away from an essentially hostile environment. The process of cytoplasmic streaming required for bleb formation must involve considerable water movement and thus is likely to be subject to osmotic changes; therefore, the expression of membrane channel proteins responsible for water transport, such as aquaporins (AQPs), is of major research interest.

AQPs are small, hydrophobic, integral transmembrane proteins whose major function is to facilitate the transport of water molecules $(16,17)$. To date, 300 distinct AQPs have been discovered in diverse organisms, with 13 mammalian isoforms having been identified (AQP0-AQP12). Based on their functional and structural properties, AQPs are classified into subfamilies: The classical AQPs, including AQP0, AQP1, AQP2, AQP4, AQP5, AQP6 and AQP8, are selective for water transport, whereas the aquaglyceroporins and the super-aquaporins, which include AQP3, AQP7, AQP9 and AQP10, also transport small solutes such as ammonia, urea and glycerol. AQP3 is selective for water and glycerol $(16,17)$.

In addition to the various physiological roles of AQPs, they have also been linked to cancer pathogenesis in various organs including the lung (18), colon (19), brain (22) and liver (21). Shi et al (16) reported the expression of several AQPs in breast cancer, including AQPs 1, 3, 4 and 5; all were upregulated in cancer tissue apart from AQP4, which was more intensely expressed in normal tissue. AQP1 expression was detected in basal-like (22) and luminal cancer subtypes (23), and associated with poor prognosis. AQP1 overexpression was shown to enhance breast cancer proliferation and invasion in vitro (23), while small interfering RNA (siRNA)-mediated knockdown of AQP1 decreased tumor mass and volume in vivo (24). Enhanced AQP3 expression in patients with early breast cancer was associated with poor prognosis (25). Treating ER-positive $\left(\mathrm{ER}^{+}\right)$breast cancer cells with estrogen upregulated expression of AQP3; this is likely via activation of estrogen response elements (EREs) in its gene promoter (26). Additionally, siRNA knockdown of AQP3 significantly reduced cell migration and invasion (26-28). AQP4 has been reported to be elevated in breast cancer tissues (16), and its siRNA-mediated downregulation in $\mathrm{ER}^{+}$breast cancer cells enhanced the expression of E-cadherin, and decreased cell proliferation, motility and invasion (29). Enhanced expression of AQP5 in tumors has been associated with poor clinical prognosis and lymph node metastasis (30), and shRNA-knockdown of AQP5 in MCF-7 cells significantly decreased cell proliferation and migration (31).

In the present study, it was determined that alkaline $\mathrm{pH}$ induces bleb formation in endocrine-resistant pII breast cancer cells, regulated in part by water movement in response to osmotic changes in the extracellular environment. AQP3 (and to a certain extent, AQP1) was translocated to newly formed blebs. In addition, siRNA-mediated knockdown of
AQP3 in pII cells significantly decreased bleb formation, and cell motility and invasion. These data suggested that AQP3 may play a role in bleb formation/stabilization, and thereby help promote invasion and metastasis.

\section{Materials and methods}

Cell lines. The ER-negative (ER') MDA-MB-231 human breast carcinoma cell line was originally obtained from the American Type Culture Collection (ATCC). MCF10A normal breast epithelial cells were obtained from Dr Elizabeth Saunderson through Dr Jenny Gomm (St Bartholomew's Hospital, London). pII (ER') cells were generated via shRNA-mediated knockdown of ER in MCF7 cells (which were also originally obtained from the ATCC); YS1.2 was derived from similarly transfected MCF7 cells, in which ER was not downregulated; therefore, this cell line is used as $\mathrm{ER}^{+}$control for pII $(8,11)$. For routine culture, all cell lines were maintained as monolayers at $37^{\circ} \mathrm{C}$ in an incubator with an atmosphere of $5 \% \mathrm{CO}_{2}$ at $95 \%$ humidity, in advanced DMEM containing phenol red as a pH indicator and supplemented with 5\% FBS, $600 \mu \mathrm{g} / \mathrm{ml}$ L-glutamine, $100 \mathrm{U} / \mathrm{ml}$ penicillin, $100 \mu \mathrm{g} / \mathrm{ml}$ streptomycin and $6 \mathrm{ml} / 500 \mathrm{ml}$ 100X non-essential amino acids (all from Invitrogen; Thermo Fisher Scientific, Inc.).

Morphology of pII cells exposed to alkaline $\mathrm{pH}$ and osmotic changes. Cells were removed from the $\mathrm{CO}_{2}$ incubator and exposed to standard atmospheric conditions, which caused the medium $\mathrm{pH}$ to rise to 8.3 (13) within 10-30 min (depending on whether grown in culture plates or flasks). The morphology of cells under control ( $\mathrm{pH}$ 7.4) and alkaline ( $\mathrm{pH}$ 8.3) conditions was monitored via phase contrast light microscopy. Images were captured for the same field at 0 and $30 \mathrm{~min}$ (4 fields for each condition; magnification, $\mathrm{x} 40$ ). The number of blebbing cells was counted manually to compare the percentage of cells blebbing. Live cell microscopy with time-lapse photography was used to continuously monitor the effect of osmotic changes on the bleb morphology of pII cells. For this, cells were grown in a $10-\mathrm{mm}$ culture dish containing $2 \mathrm{ml}$ DMEM and exposed to standard atmospheric conditions for $30 \mathrm{~min}$ to alkalinize the medium. The cells were then placed inside an imaging chamber (Cell Observer HS; Carl Zeiss AG) heated with an airstream to $37^{\circ} \mathrm{C}$ with normal atmosphere and monitored for $120 \mathrm{~min}$. Cells were photographed at x40 magnification and images were captured at 5-min intervals. This experiment was repeated by first inducing alkaline conditions for $30 \mathrm{~min}$, followed by the removal of the original media, and substitution with media diluted with water (1:1 and 1:4) or sucrose solution (1-100 $\mathrm{mM}$ ) for $30 \mathrm{~min}$ to produce acute hypotonic or hypertonic conditions, respectively.

Reverse transcription-quantitative PCR (RT-qPCR). RNA was extracted from cell pellets using an RNeasy kit from Qiagen, Inc., and $2 \mu \mathrm{g}$ (in $20 \mu \mathrm{l}$ reaction mix) was converted to cDNA using a High Capacity cDNA Reverse Transcriptase kit from Applied Biosystems (Thermo Fisher Scientific, Inc.). qPCR was performed on $1 \mu \mathrm{l}$ of the cDNA reaction mix using a standard multiplexed TaqMan PCR kit protocol (Applied Biosystems; Thermo Fisher Scientific, Inc.) to determine the expression of AQPs. Control gene probes (human $\beta$-actin; cat. no. 4310881E; 
Thermo Fisher Scientific, Inc.) were labeled with VIC ${ }^{\mathrm{TM}}$, and target gene probes for AQP1 (cat. no. Hs01028916_m1), AQP3 (cat. no. Hs00185020_m1), AQP4 (cat. no. Hs00242342_m1) and AQP5 (cat. no. Hs00387048_m1; all Applied Biosystems; Thermo Fisher Scientific, Inc.) were labeled with fluorescein amidite. The amplifications were performed on an Applied Biosystems 7500 HT Fast thermocycler under the following conditions: $95^{\circ} \mathrm{C}$ for $10 \mathrm{~min}$, followed by 40 cycles of $95^{\circ} \mathrm{C}$ for $15 \mathrm{sec}$ and $60^{\circ} \mathrm{C}$ for $1 \mathrm{~min}$. The raw quantification cycle $(\mathrm{Cq})$ values were converted via the $\Delta \Delta \mathrm{Cq}$ method to determine normalized expression ratios of the target genes relative to $\beta$-actin (32).

Western blotting. Cells were cultured in 6-well plates with complete DMEM to 80-90\% confluence. Medium was subsequently aspirated off, and cell monolayers were harvested by scraping and resuspended into $300 \mu \mathrm{l}$ of lysis buffer containing $50 \mathrm{mM}$ HEPES, $50 \mathrm{mM} \mathrm{NaCl}, 5 \mathrm{mM}$ EDTA 1\% Triton $\mathrm{X}$, $100 \mu \mathrm{g} / \mathrm{ml}$ PMSF, $10 \mu \mathrm{g} / \mathrm{ml}$ aprotinin and $10 \mu \mathrm{g} / \mathrm{ml}$ leupeptin. Protein concentration was determined via the Bradford assay using BSA (Sigma-Aldrich; Merck KGaA) as standard, and $8 \mu \mathrm{g}$ protein lysate was mixed with an equal volume of $2 \mathrm{X}$ SDS and heated at $90^{\circ} \mathrm{C}$ for $10 \mathrm{~min}$. Samples were loaded onto a $10 \%$ SDS-polyacrylamide gel and electrophoresed at $150 \mathrm{~V}$ for $1 \mathrm{~h}$. Proteins were transferred to a PVDF membrane and blocked with $2 \% \mathrm{BSA}$ for $1 \mathrm{~h}$ at $4^{\circ} \mathrm{C}$ before being incubated overnight at $4^{\circ} \mathrm{C}$ with AQP3 antibody (1:500; cat. no. ab125219; Abcam) prepared in $2 \%$ BSA. The membrane was washed and incubated with anti-HRP-conjugated secondary antibody (1:500; cat. no. 7074; Cell Signaling Technology, Inc.) for $1 \mathrm{~h}$ at $4^{\circ} \mathrm{C}$, developed with Super Signal ECL (Thermo Fisher Scientific, Inc.) and visualized with Kodak X-ray film. Blots were also probed with $\beta$-actin antibody (1:1,000; cat. no. 8457; Cell Signaling Technology, Inc.) as a loading control.

Immunofluorescence analysis. Cells were seeded at $\sim 5,000$ cells/well in an 8 -well chambered slide containing $300 \mu \mathrm{l} /$ well DMEM, and left to grow under standard culture conditions for 2 days. pII cells were also cultured under standard conditions for 2 days before subsequent exposure to alkaline $\mathrm{pH}$ conditions for $30 \mathrm{~min}$. Media were removed, and cells were immediately fixed by addition of $3.7 \%$ formaldehyde $(200 \mu \mathrm{l} /$ well) for $10 \mathrm{~min}$ with gentle agitation at room temperature. Alternatively, cells were fixed by incubation with ice-cold $100 \%$ methanol for $15 \mathrm{~min}$ at $-20^{\circ} \mathrm{C}$. Removal of the fixative was followed by three washing steps with ice-cold PBS, each for $5 \mathrm{~min}$. Non-specific binding sites were blocked by addition of $1 \%$ BSA in PBS $(100 \mu \mathrm{l})$, and cells were incubated at room temperature for $1 \mathrm{~h}$. The BSA was then aspirated, and $200 \mu \mathrm{l} /$ well of primary antibody solution was added and left to incubate overnight at $4^{\circ} \mathrm{C}$, with anti-AQP1 (cat. no. ab11025), anti-AQP3 (cat. no. ab125219), anti-AQP4 (cat. no. ab46182) or anti-AQP5 (cat. no. ab15119) antibodies added (all 1:100; all Abcam). Following removal of the primary antibodies, cells were washed as aforementioned and the secondary antibody (Alexa Fluor ${ }^{\circledR}$ 555-conjugated anti-rabbit IgG; 1:500; cat. no. 4413; Cell Signaling Technology, Inc.) was added for a further 2 -h incubation at room temperature in the dark. This was followed by a repeat of the previously described washing steps after removal of the antibody solution.
Then, $200 \mu \mathrm{l} /$ well of diluted phalloidin (6 $\mu \mathrm{l}$ in $1 \mathrm{ml} \mathrm{PBS}$; Thermo Fisher Scientific, Inc.) was added and incubated for $10 \mathrm{~min}$ at room temperature in the dark. After removing the phalloidin and washing with PBS three times, the chambers and borders of the 8-well chambered slides were removed, and a drop of DAPI was added onto the slide immediately prior to mounting. Staining was visualized and photographed using an LSM 510 Meta confocal microscope (Carl Zeiss AG) at an excitation wavelength of $450 \mathrm{~nm}$.

For comparison, experiments were also performed using AQP antibodies sourced from Biorbyt Ltd. [AQP1 (cat. no. orb10122), AQP3 (cat. no. orb47955), AQP4 (cat. no. orb10125) and AQP5 (cat. no. orb395791); all 1:100) and Santa Cruz Biotechnology, Inc. [AQP1 (cat. no. sc-25287), AQP3 (cat. no. sc-518001), AQP4 (cat. no. sc-32739) and AQP5 (cat. no. sc-514022); all 1:100; data not shown].

AQP3 siRNA transfection. Cells were plated in 12-well plates in complete DMEM, incubated for $24 \mathrm{~h}$ at $37^{\circ} \mathrm{C}$ with $5 \% \mathrm{CO}_{2}$, and allowed to grow until reaching 70-90\% confluence. Transfection was then performed using control scramble siRNA (cat. no. sc-37007) or AQP3 siRNA (cat. no. sc-29713; both Santa Cruz Biotechnology, Inc.). Solution A was prepared by adding $6 \mu \mathrm{l}$ Lipofectamine ${ }^{\circledR}$ RNAiMAX transfection reagent (cat. no. 56532; Invitrogen; Thermo Fisher Scientific, Inc.) to $100 \mu \mathrm{l}$ of Opti-MEM reduced serum medium (Invitrogen; Thermo Fisher Scientific, Inc.), and solution B was prepared by diluting $2 \mu \mathrm{l}$ of AQP3 siRNA into $100 \mu \mathrm{l}$ of Opti-MEM reduced serum medium $(0.2 \mu \mathrm{M}$ final concentration). Solutions A and B were mixed, incubated for $15 \mathrm{~min}$ and added dropwise to the cells. Cells were then incubated at $37^{\circ} \mathrm{C}$ with $5 \% \mathrm{CO}_{2}$. Following 48-72-h incubation, cells were harvested and RNA was extracted for the determination of AQP3 expression via RT-qPCR. Other transfected cells were harvested at $72 \mathrm{~h}$ for AQP3 protein determination via western blotting, or after $48 \mathrm{~h}$ for motility and invasion assays, as well as determining the effects of alkaline $\mathrm{pH}$ on cell morphology via immunofluorescence confocal microscopy.

Motility assay. Cells (control and following AQP3 siRNA transfection for $48 \mathrm{~h}$ ) were cultured in 12-well plates with complete DMEM containing 5\% FBS to 80-90\% confluence. A scratch was created in the cell monolayer using a sterile P1000 pipette tip, and a photograph of the scratched area was captured immediately $(0 \mathrm{~h})$. After overnight incubation, another photograph (both magnification, x10) was captured of the same scratched area. The width of the scratch at $24 \mathrm{~h}$ was calculated as a percentage of the width at $0 \mathrm{~h}$; a minimum of three areas along the scratch were measured.

Cultrex basement membrane extract (BME) cell invasion assay. Cell invasion was assessed using a Cultrex 24-well BME cell invasion assay purchased from Trevigen, Inc. (cat. no. 3455-024-K) according to the manufacturer's instructions. For this, the invasion chamber was coated with $100 \mu \mathrm{l}$ of 1X BME solution and incubated overnight at $37^{\circ} \mathrm{C}$. After $24 \mathrm{~h}$ of transfection, cells were serum-starved overnight at $37^{\circ} \mathrm{C}$ with $5 \% \mathrm{CO}_{2}$. On the following day (after $48 \mathrm{~h}$ of transfection), pII cells were harvested, counted and diluted to $1 \times 10^{6}$ cells $/ \mathrm{ml}$ in serum-free medium. After that, $100 \mu \mathrm{l}$ of cells were added 
A
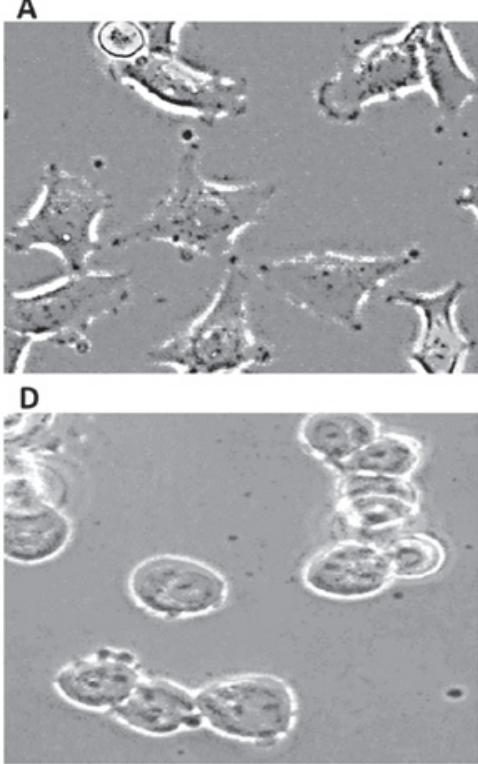

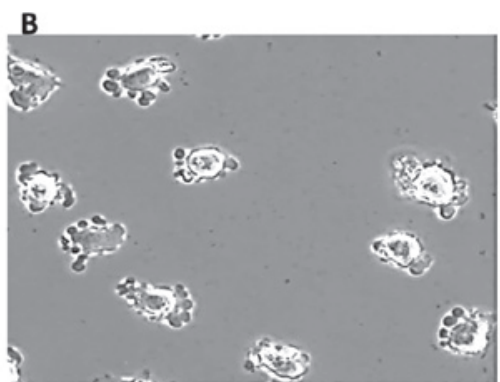

E

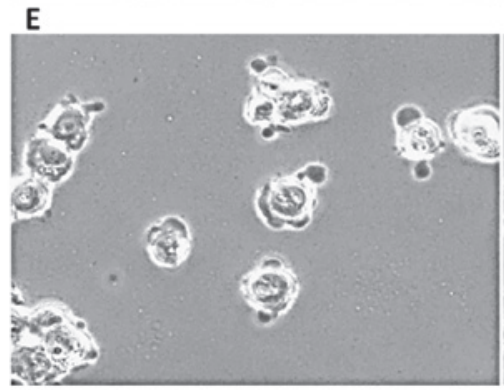

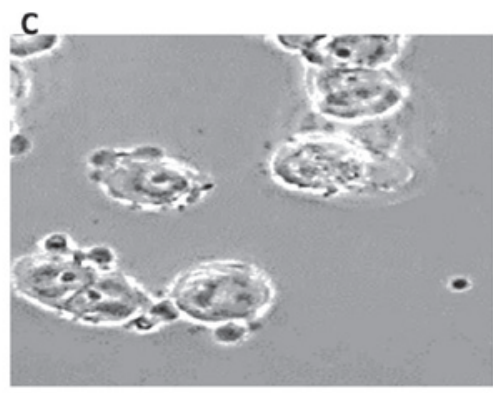

F

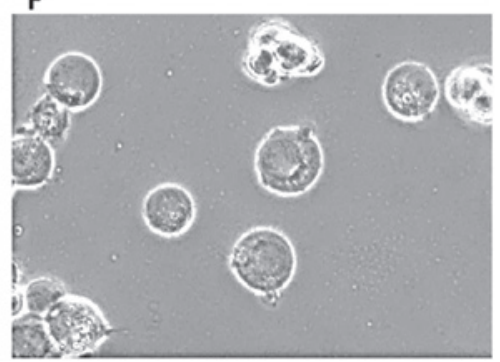

Figure 1. Effect of hypotonic conditions on alkaline $\mathrm{pH}$-induced bleb formation in pII cells. Representative images of pII cells (grown in 10-mm culture dishes in DMEM at $37^{\circ} \mathrm{C}$ and $5 \% \mathrm{CO}_{2}$ ) taken by phase contrast light microscopy (A) immediately after removal from incubator at pH 7.4 or (B) after exposure to alkaline conditions ( $\mathrm{pH} \mathrm{8.3)} \mathrm{for} 30 \mathrm{~min}$. In a separate experimental setup, cells were first exposed to alkaline pH for $30 \mathrm{~min}$ until they formed blebs, and the media was then removed and replaced with a solution composed of (C) DMEM diluted with water (1:1) for 30 min, (D) diluted 1:1 for 60 min, (E) diluted 1:4 for $30 \mathrm{~min}$ or $(\mathrm{F})$ diluted 1:4 for $60 \mathrm{~min}$. Cells were observed at $\mathrm{x} 40$ magnification in a live cell imager heated to $37^{\circ} \mathrm{C}$ with an airstream under normal atmospheric conditions.

to the top chamber of the Cultrex dish. The lower chamber was loaded with $500 \mu \mathrm{l}$ of DMEM supplemented with $30 \%$ FBS (used as a chemoattractant). Cells were incubated at $37^{\circ} \mathrm{C}$ with $5 \% \mathrm{CO}_{2}$ and allowed to invade to the bottom chamber. After $24 \mathrm{~h}$, the top and the bottom chambers were aspirated and washed with $1 \mathrm{X}$ cell wash buffer. Calcein-AM/cell dissociation solution complex was added to the bottom chamber and left for $1 \mathrm{~h}$ at $37^{\circ} \mathrm{C}$ with $5 \% \mathrm{CO}_{2}$. Cells internalize calcein-acetomethylester (AM), and intracellular esterases cleave the AM moiety, generating fluorescence-free calcein. Invading cells were determined by recording the fluorescence emission using a microplate reader, with an excitation/emission filter set of $485 / 535 \mathrm{~nm}$.

Statistical analysis. Data are presented as the mean \pm SEM of three independent experiments, and were analyzed using GraphPad Prism 5 software (GraphPad Software, Inc.). Student's two-tailed unpaired t-test or one-way ANOVA followed by Bonferroni post hoc test were used to compare means of individual groups. $\mathrm{P}<0.05$ was considered to indicate a statistically significant difference.

\section{Results}

Effects of alkaline $\mathrm{pH}$ and hypotonic/hypertonic conditions in the extracellular environment on the morphology and bleb formation of pII cells. Changes in the morphology of pII cells were recorded by photographing immediately after removal from the incubator at pH 7.4 (control; Fig. 1A), and then again after $30 \mathrm{~min}$ exposure to atmospheric conditions (pH 8.3; Fig. 1B). From their typical mesenchymal-like spindle morphology, the cells became rounded and exhibited extensive blebbing on the plasma membrane. To determine the effect of osmotic changes on $\mathrm{pH}$-induced bleb formation, pII cells were first exposed to alkaline conditions for $30 \mathrm{~min}$ (to form the blebs) followed by the removal of the original media and substitution with media diluted with water to produce acute hypotonic conditions. As shown in Fig. 1C-F, substitution with DMEM diluted $1: 1$ or 1:4 with water for 30-60 min caused the cells to become more rounded and enlarged in size, and after $60 \mathrm{~min}$ (Fig. 1D and F), the blebs had almost entirely disappeared. Notably, the cells were able to withstand severe hypotonic conditions without lysing even after exposure for $120 \mathrm{~min}$ (data not shown). Prior hypotonic conditions caused the cells to swell; this prevented bleb formation when the medium $\mathrm{pH}$ was allowed to alkalinize (data not shown).

In another experimental setup, pII cells were first exposed to alkaline $\mathrm{pH}$ conditions (Fig. 2A) followed by the addition of sucrose solution to the media at various concentrations $(1,10$ and $100 \mathrm{mM}$ ) for $30 \mathrm{~min}$ (Fig. 2B-D, respectively) to induce acute hypertonic conditions. The cells became rounded and the blebs almost entirely disappeared. These data suggested that water movement across the plasma membrane is essential for alkaline $\mathrm{pH}$-induced bleb formation in pII cells.

Expression profile of various AQPs in breast epithelial and cancer cell lines. RT-qPCR analysis showed that AQP1 was expressed at very low levels in MCF10A and non-invasive YS1.2 cells (12), but its levels were significantly higher in the ER- breast cancer cells (Fig. 3A). AQP3 was highly expressed in YS1.2, followed by pII and MDA-MB-231, with the lowest expression in MCF10A cells (Fig. 3B). AQP4 was mainly expressed in MCF10A, with very low expression in breast cancer cells (Fig. 3C). Similar to AQP1 and AQP3, AQP5 was highly expressed in breast cancer cells compared with MCF10A (Fig. 3D). Western blotting was used to confirm the 

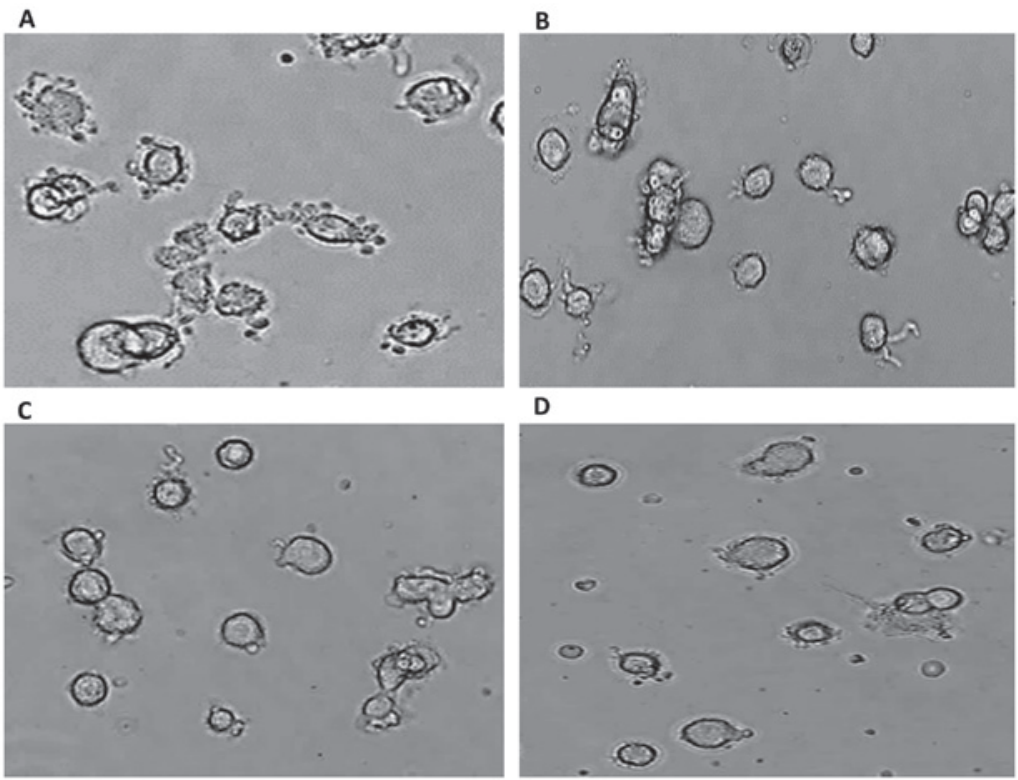

Figure 2. Effect of hypertonic conditions on alkaline pH-induced bleb formation in pII cells. (A) Representative image of pII cells (grown in 10-mm culture dishes in DMEM at $37^{\circ} \mathrm{C}$ and $5 \% \mathrm{CO}_{2}$ ) taken by phase contrast light microscopy after exposure to alkaline conditions for 30 min. In another experimental setup, cells were first exposed to alkaline $\mathrm{pH}$ for $30 \mathrm{~min}$ until they formed blebs, and the media was then removed and replaced with a solution composed of (B) $1 \mathrm{mM}$, (C) $10 \mathrm{mM}$ or (D) $100 \mathrm{mM}$ sucrose for $60 \mathrm{~min}$. Cells were observed at $\mathrm{x} 40$ magnification in a live cell imager heated to $37^{\circ} \mathrm{C}$ with an airstream under normal atmospheric conditions.
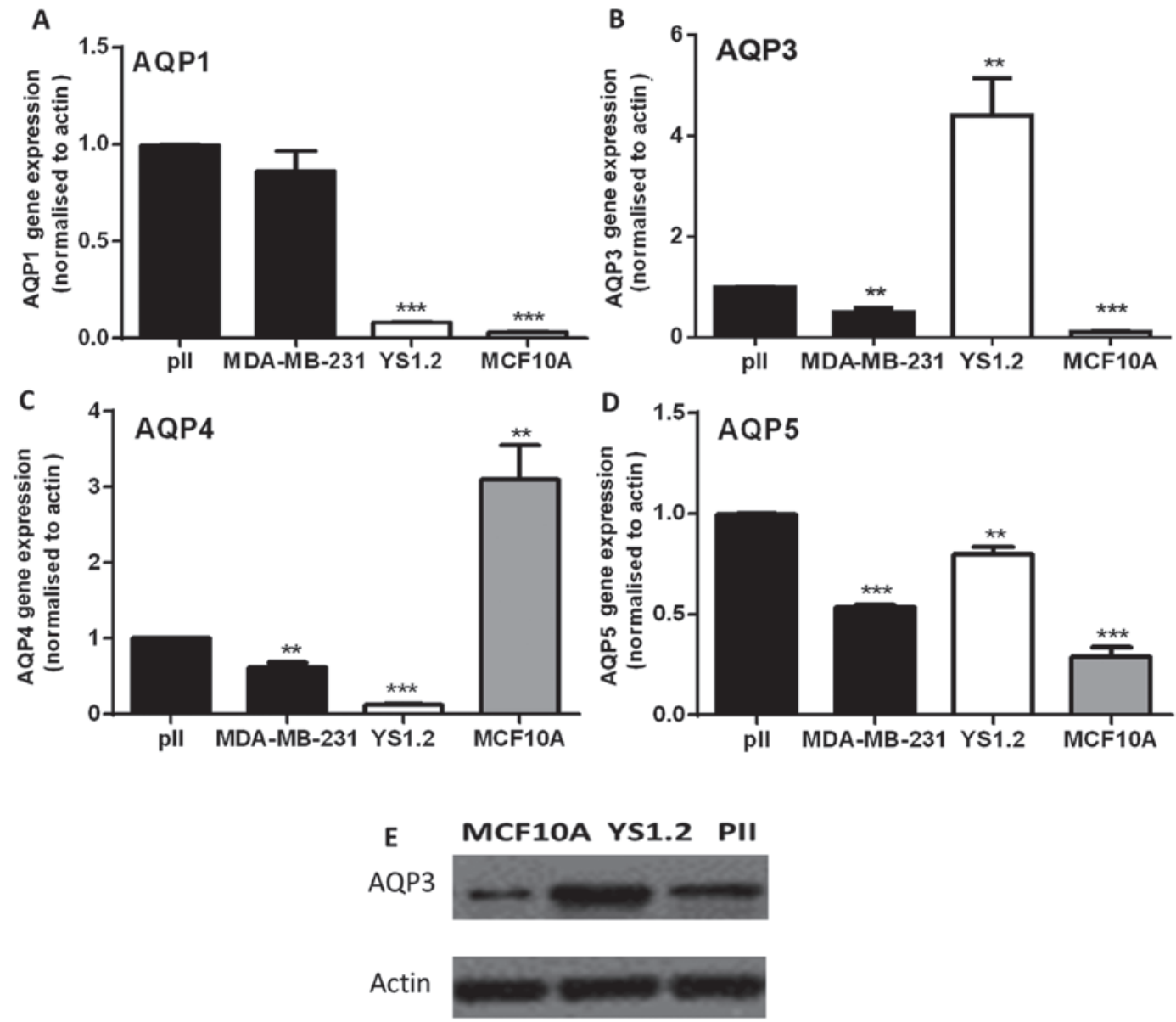

Figure 3. Expression of AQPs in normal breast MCF10A cells and breast cancer cell lines. RNA extracted from cell pellets and converted to cDNA was analyzed via reverse transcription-quantitative PCR to determine the expression of (A) AQP1, (B) AQP3, (C) AQP4 and (D) AQP4 (normalized to actin). The expression for each cell line was calculated based on quantification cycle values as a normalized ratio to the expression level observed in pII cells. (E) Western blot analysis of AQP3 protein expression. Cell lysates were separated via 10\% SDS-PAGE, blotted onto PVDF membranes and probed with antibodies targeted against $\mathrm{AQP} 3$ and $\beta$-actin; the presented blot is representative of 3 independent experiments. Data are presented as the mean \pm SEM of 3 independent experiments. ${ }^{* * *} \mathrm{P}<0.01,{ }^{* * *} \mathrm{P}<0.001$ vs. pII. AQP, aquaporin. 

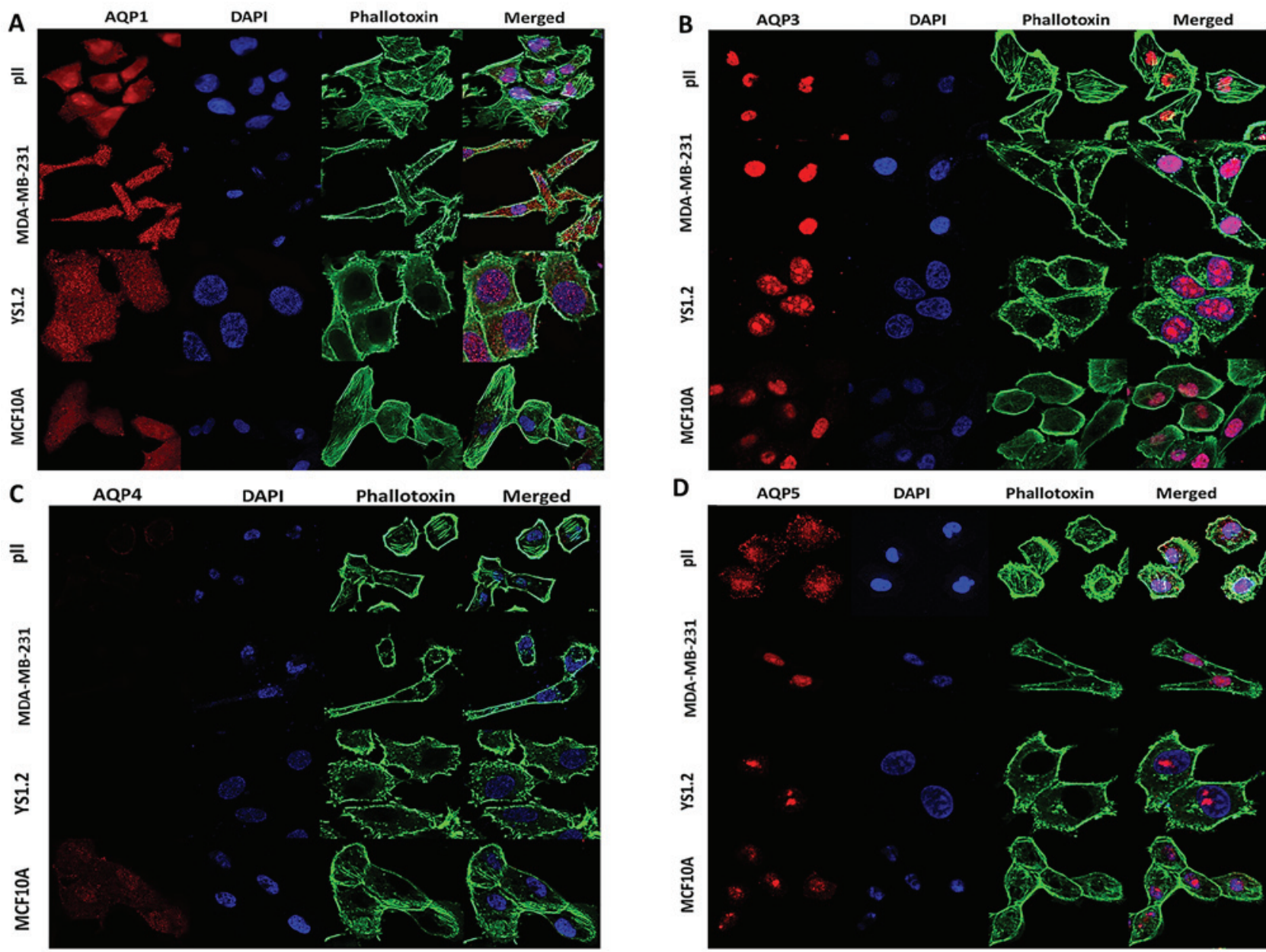

Figure 4. Immunolocalization of various AQPs in breast cell lines. Cells were grown in 10 -mm culture dishes in DMEM at $37^{\circ} \mathrm{C}$ and $5 \% \mathrm{CO}_{2}$ at $\mathrm{pH} 7.4(\mathrm{control})$ and stained with (A) AQP1, (B) AQP3, (C) AQP4 and (D) AQP5 antibodies (red), as well as phalloidin (green) and DAPI (blue), before being imaged under a confocal microscope. Magnification, $\mathrm{x} 60 . \mathrm{AQP}$, aquaporin

expression profile of AQP3 at the protein level in MCF10A, YS1.2 and pII cells (Fig. 3E). These results are consistent with the gene expression profile, which shows differing expression between $\mathrm{ER}^{-}$and $\mathrm{ER}^{+}$breast cancer cell lines. In general, AQP3 expression was higher in cancerous compared to non-cancerous breast cells. The gene expression profile of the tested AQPs was not modified in pII cells upon exposure to alkaline $\mathrm{pH}$ for $30 \mathrm{~min}$ (data not shown).

Localization pattern of various AQPs at normal $\mathrm{pH}$ and upon exposure to alkaline $\mathrm{pH}$. The localization patterns of AQPs 1 , 3,4 and 5 in the tested cell lines cultured in $\mathrm{pH} 7.4$ were determined via immunofluorescence. Due to differences in microscope settings and digital enhancement of certain images (necessary to visualise low expression), fluorescence intensities presented in the figures do not accurately reflect relative quantitative differences. AQP1 showed diffuse cytoplasmic staining in all the cell lines examined, as well as expression over the nuclear surface; immunoreactivity was weakest in MCF10A cells, confirming comparatively low levels of expression of this protein in normal breast epithelial cells (Fig. 4A). Staining with an anti-AQP3 antibody from Abcam, expression was detected only in the nuclear region, with no clear staining in the cytoplasm (Fig. 4B); this pattern was observed in all the cell lines. In view of this somewhat unexpected distribution of AQP3 compared with other literature reports $(28,33)$, these experiments were repeated in pII cells with antibodies purchased from other sources, which showed similar nuclear localization (data not shown). Additionally, an alternative fixation method using methanol instead of formaldehyde was used to determine whether this affected the interaction of the antibody, and similar distribution patterns were also observed (data not shown). Weak diffuse staining of AQP4 was observed only in MCF10A (Fig. 4C). AQP5 appeared to be mainly nuclear in all the cell lines, with some additional cytoplasmic immunoreactivity detected in pII cells (Fig. 4D).

The locations of AQPs 1, 3, 4 and 5 were also determined in $\mathrm{pII}$ cells following exposure to alkaline $\mathrm{pH}$ for $30 \mathrm{~min}$. As presented in Fig. 5A, AQP1 exhibited a similar diffuse distribution in alkaline $\mathrm{pH}$, but was now also detected inside the blebs. AQP3 remained predominantly nuclear but had also markedly migrated into the blebs, in a far more pronounced manner than AQP1 (Fig. 5B). AQP4 was not detectable at either pH (Fig. 5C). AQP5 maintained its nuclear localization, and was not observed in either the extranuclear space or inside the blebs (Fig. 5D). These data suggested that AQP3 may play an important role in pII cell migration and invasion due to its substantial translocation into the newly formed blebs. 


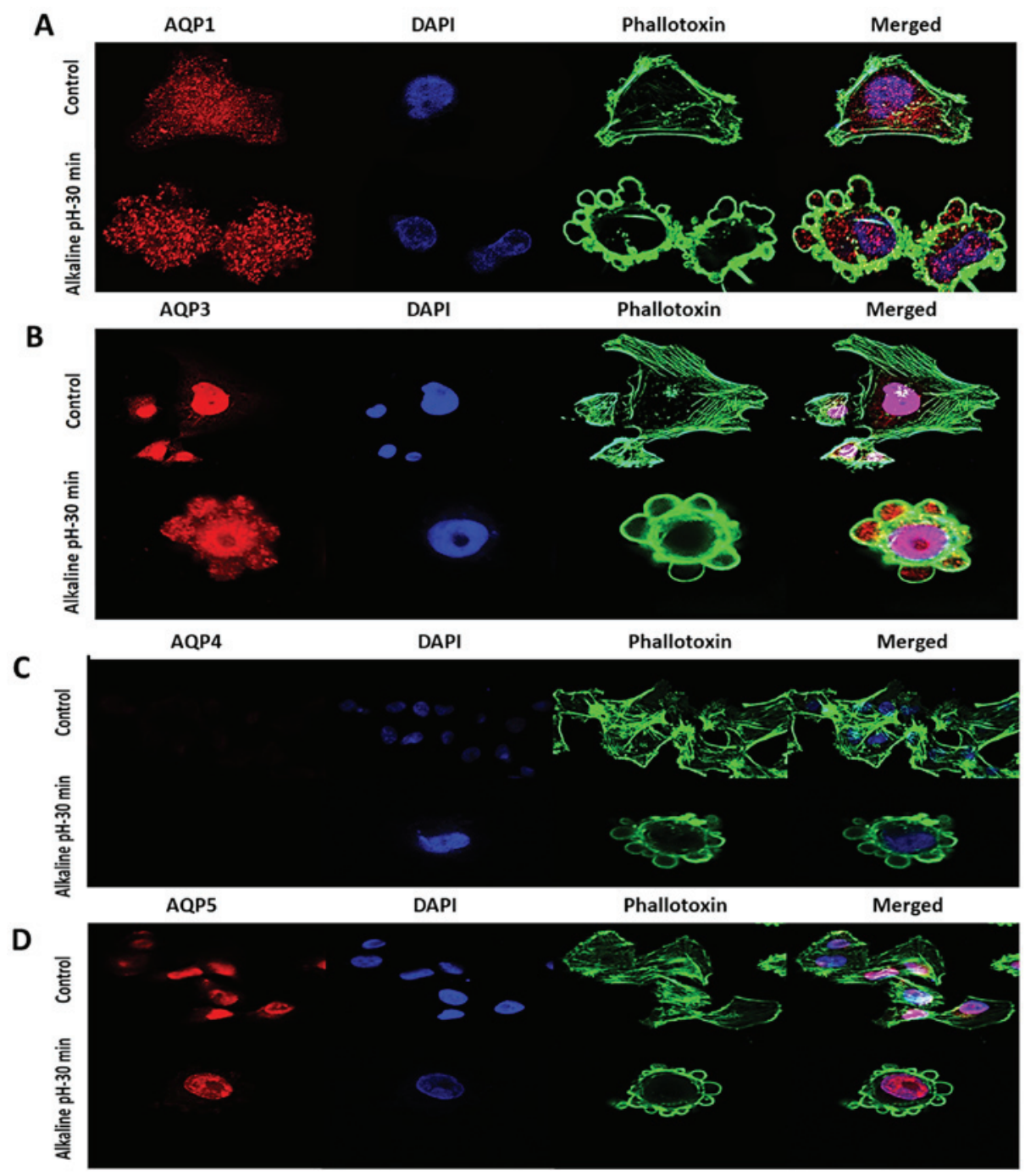

Figure 5. Effect of exposure to alkaline $\mathrm{pH}$ on the localization of AQPs in pII cells. pII cells were cultured in $10-\mathrm{mm}$ dishes in $\mathrm{DMEM}^{2} 37^{\circ} \mathrm{C}$ and $5 \% \mathrm{CO}_{2}$ at $\mathrm{pH} 7.4$ (control), or exposed to $\mathrm{pH} 8.3$ for 30 min, and subsequently stained with (A) AQP1, (B) AQP3, (C) AQP4 and (D) AQP5 antibodies (red), as well as phalloidin (green stain) and DAPI (blue stain), before being imaged under a confocal microscope. Magnification, x60. AQP, aquaporin.

Effects of siRNA knockdown of AQP3 on pII cell blebbing, motility and invasion. AQP3 gene expression was significantly reduced in pII cells at 48 and $72 \mathrm{~h}$ post-transfection with AQP3-targeting siRNA (Fig. 6A). This was paralleled by a reduction in AQP3 protein detected via western blotting at $72 \mathrm{~h}$ (Fig. 6B). Cultures assayed at $48 \mathrm{~h}$ post-transfection exhibited significant reduction in cell motility (Fig. 6C and D) and invasion towards serum components (Fig. 6E). It should be noted that the motility assays were performed in media supplemented with 5\% FBS over $24 \mathrm{~h}$. This is expected to result in doubling of the cell number; however, the contribution of cell proliferation to total wound closure is expected to be relatively minor. This can be clearly demonstrated by the difference between migratory and non-migratory cells cultured in growth media. Assessment of the proportion of blebbing cells in siRNA-transfected cultures exposed to alkaline $\mathrm{pH}$ also showed a significant reduction (Fig. 6F). The immunofluorescence images presented in Fig. 6 G show examples of the heterogeneity of response in individual cells; the left panel shows AQP3 expression in control (scrambled siRNA)-transfected blebbing cells, whereas the right panels show AQP3 siRNA-transfected cells that either failed to bleb, or blebbed despite the absence of AQP3.

\section{Discussion}

The present study revealed distinct expression profiles for AQPs 1, 3, 4 and 5 in normal epithelial breast cells and breast cancer cells. mRNA expression levels of AQPs 1, 3 and 5 were higher in breast cancer cells compared with MCF10A. Conversely, AQP4 expression was higher in MCF10A than in the cancer cells. These results are in agreement with a number of reports which demonstrated enhanced AQP 1, 3 and 5 expression in breast cancer tissues $(16,22,31)$, particularly in those with invasive ductal carcinoma and lymph node invasion (for AQP5) (31). AQP1 expression was found to be correlated with high tumor grade and triple negativity, and exhibited a negative relationship with ER status (22). This inverse correlation is in agreement with the present findings into AQP1 mRNA expression. In contrast, the expression of AQP3 was elevated in $\mathrm{ER}^{+}$breast cancer tissues obtained from premenopausal women in comparison to those from postmenopausal 

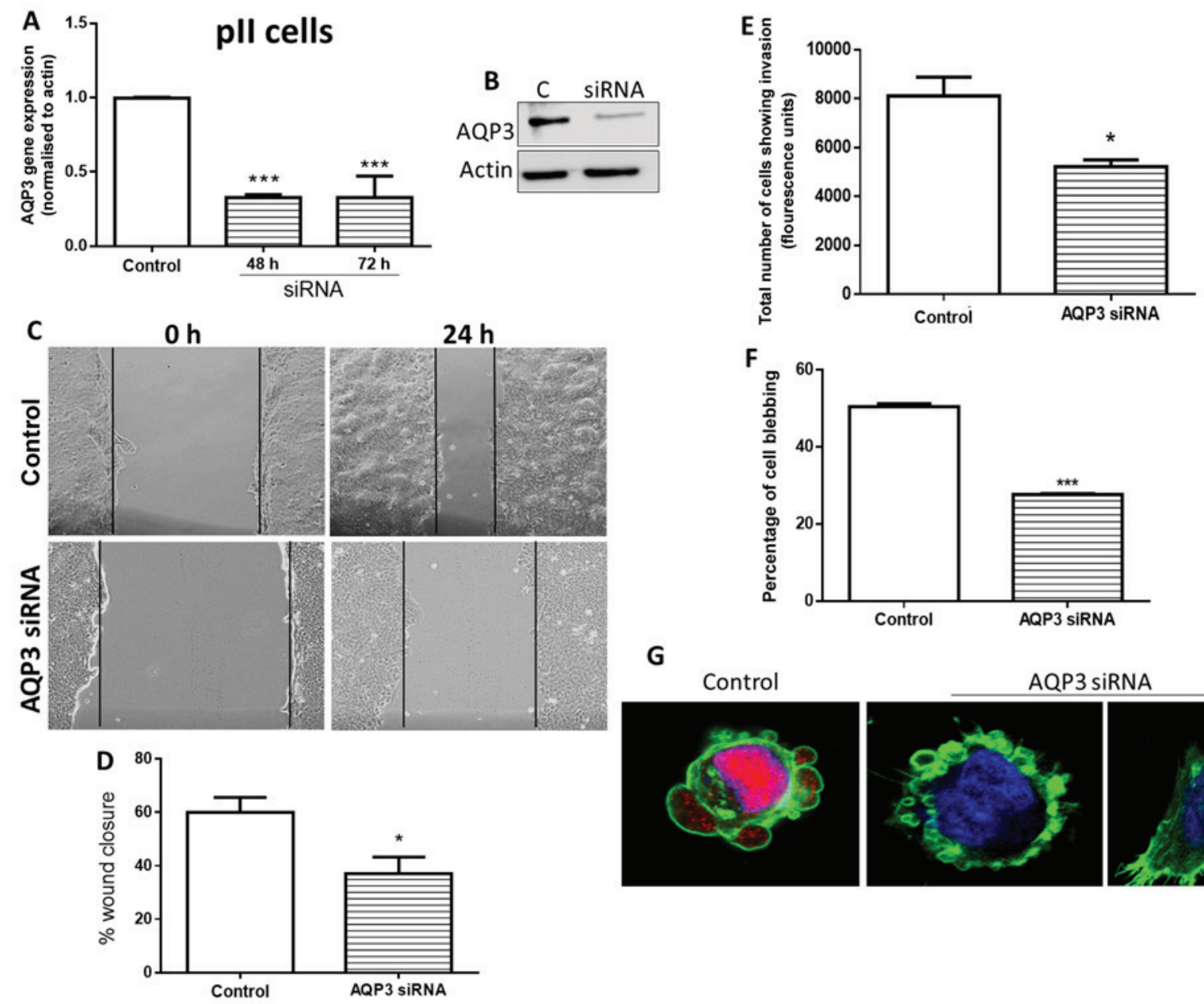

$\mathbf{G}$
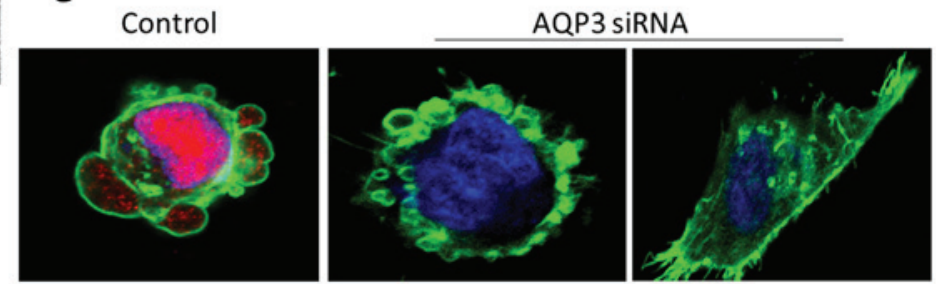

Figure 6. Effects of siRNA-mediated knockdown of AQP3 in pII cells on cell motility, invasion and alkaline pH-induced bleb formation. (A) Reverse transcription-quantitative PCR analysis of AQP3 mRNA expression in control siRNA-transfected cells, and AQP3 siRNA-transfected cells at 48 and $72 \mathrm{~h}$. (B) AQP3 protein expression was determined in control siRNA- and AQP3 siRNA-transfected cells at $72 \mathrm{~h}$ via western blot analysis. (C) At $48 \mathrm{~h}$ following transfection, cells were grown to confluency, and a scratch was made through the center of the cell monolayer using a $1000 \mu 1$ Eppendorf pipette. The scratch width was measured under a microscope at 0 and $24 \mathrm{~h}$. (D) Percentage of wound closure was quantified. (E) Effect of AQP3 siRNA knockdown on invasion of pII cells through basement membrane extract towards serum components. The y-axis represents arbitrary fluorescence units showing uptake of calcein into the invading cells in the bottom chamber of the Cultrex dish. (F) At $48 \mathrm{~h}$ after transfection with AQP3 siRNA, cells were removed from the incubator and exposed to atmospheric conditions. Cells were photographed immediately ( $\mathrm{pH} \mathrm{7.4,} \mathrm{control)} \mathrm{and} \mathrm{after} 30 \mathrm{~min}(\mathrm{pH} 8.3)$. The number of blebbing cells was counted manually to compare the percentage of cells blebbing between transfected and untreated pII cells. (G) Confocal images of AQP3/phalloidin/DAPI staining of individual cells in control siRNA- and AQP3 siRNA-transfected cultures exposed to alkaline $\mathrm{pH}$ (magnification, $\mathrm{x} 60$ ). Data are presented as the mean $\pm \mathrm{SEM}$ of 3 independent experiments. ${ }^{*} \mathrm{P}<0.05,{ }^{* * *} \mathrm{P}<0.001$ vs. control. AQP, aquaporin; siRNA, small interfering RNA.

women (33). Additionally, treating $\mathrm{ER}^{+}$(T47D and MCF7) cells and ER ${ }^{-}$(MDA-MB-231) cells with estrogen enhanced AQP3 mRNA levels only in the $\mathrm{ER}^{+}$cells (26), which was attributed to the presence of an ERE in the promoter region of the AQP3 gene in the ER ${ }^{+}$breast cancer cells. This observation was also in agreement with the present findings regarding AQP3 expression.

Immunocytochemical analysis showed that AQPs 1, 3, 4 and 5 exhibited distinct cellular localization. AQP1 was evenly distributed along the cytoplasm and surrounding the nucleus. AQP3 and AQP5 were localized predominantly in the nuclear region of all cell lines, except in pII cells, which showed some cytoplasmic staining for AQP5. In contrast, AQP4 was most strongly expressed in MCF10A cells, with low expression in breast cancer cell lines. The observed cellular distribution contrasts with published reports that have generally shown the AQPs to be located in the cytoplasm or cell membrane. For example, a study conducted by Luo et al (34) demonstrated immunohistochemical localization of AQP1 in the cell membrane of breast cancer tissues, and at lower levels in the corresponding normal tissues. In their clinical study, they showed strong cytoplasmic AQP1 staining in the cells of invasive ductal carcinoma tissues and membranous staining in the cells of benign breast lesions (23). They further verified the cytoplasmic expression of AQP1 via in vitro analysis of primary breast cancer cells and MDA-MB-231 cells overexpressing AQP1. Shi et al (16) also detected the expression of AQPs 1, 4 and 5 in breast cancer and normal breast tissues, and found that AQPs 1 and 5 were located in the cellular membranes, whereas AQP4 exhibited membranous and cytoplasmic localization. Huang et al (26) showed that AQP3 exhibited cytoplasmic and membranous expression in the tumor cells of $\mathrm{ER}^{+}$breast cancer tissues. A recent study also investigated the cellular localization of AQPs 3 and 5 in triple negative breast cancer tissue, and found that both were expressed in the membrane and the cytoplasm (35). In 
view of these reports, the immunolocalization experiments were repeated to detect AQP3 in pII cells with antibodies from several commercial sources, including those used in the abovementioned studies, as well as using different fixation methods to determine whether this may account for the disparity of these observations. The results obtained with antibodies from 3 different companies all consistently confirmed the nuclear localization of AQP3 in pII cells. AQP1 was the only AQP to exhibit consistent cytoplasmic localization; AQP4 and AQP5 showed some cytoplasmic and membranous distribution in certain cell lines. Western blot analysis using the same anti-AQP3 antibody identified a band of the expected molecular weight of $32 \mathrm{kDa}$, which supports the specificity of these antibodies.

Only the ER-silenced pII cells are affected by alkaline $\mathrm{pH}$ and exhibit blebbing. Neither normal breast cells nor $\mathrm{ER}^{+}$cells show this phenomenon. ER ${ }^{-}$MDA-MB-231 cells do undergo some blebbing, but not at comparable levels to pII cells $(13,14,36)$. Therefore, the $\mathrm{pH}$ experiments were only conducted using pII cells. At present, no other stimuli that cause blebbing without inducing apoptosis have been identified. Notably, exposure to acidic $\mathrm{pH}$ does not induce this response (14). In the current study, the alterations in the morphology of pII cells exposed to alkalinity were further verified. As the formation of the blebs involves dynamic cytoplasmic streaming, this would presumably require substantial water flux, which is generally hypothesized to be controlled by the AQPs to maintain osmolality. Thus, the effects of changing the medium of pII cells that had previously been exposed to alkaline $\mathrm{pH}$ on bleb formation were examined, with the medium replaced with culture medium diluted with various ratios of water or sucrose. As would be expected the cells enlarged with increasing hypotonicity of the medium, and the blebs disappeared. Notably, the cells did not burst even after 90-120 min of observation. The redistribution of some AQP subtypes into the cellular blebs may aid to maintain the integrity of the cell and support the cytoskeleton. Under hypertonic conditions, there was also a reduction in the blebbing. As these were only exploratory observations, it will be interesting to examine the expression and distribution of AQPs during this process in further experiments. Indeed, Monzani et al (37) demonstrated that downregulation of AQP1 in a human melanoma cell line and a human microvascular cell line altered the organization of F-actin. In addition, AQP1 was found to co-immunoprecipitate with Lin-7 in these cell lines; a model was proposed that AQP1 acts as a scaffolding protein of the plasma membrane that is involved in the organization of the cytoskeleton by interacting with the Lin-7/ $\beta$-catenin pathway (37). In the present study, only AQP1 and AQP3 were observed to be translocated into the formed blebs. The redistribution of these proteins into the blebs suggests their involvement in the process of bleb formation and/or stabilization and, by implication, in cell migration and invasion. The process of cell migration has been associated with membrane protrusions such as lamellipodia and ruffling at the leading edge of migrating cells (38). $\mathrm{Hu}$ and Verkman (39) reported that AQP1 showed polarized distribution at the leading edge of migrating mouse melanoma B16F10 cells and was associated with increased migration. In the present study, siRNA-mediated knockdown of AQP3 in pII cells resulted in a significant reduction in cell migration and invasion through BME; the percentage of blebbing cells was also significantly reduced upon subsequent exposure to alkaline $\mathrm{pH}$. It should be noted that a proportion of the transfected cells still blebbed despite loss of AQP3. This is not unexpected, as our previous studies showed that several other molecules and signaling pathways are involved in the complex process of blebbing in pII cells $(13,14,40)$; these would be unaffected by AQP3 knockdown.

A number of studies have suggested that AQP3 is associated with cancer progression and metastasis in esophageal, colorectal, hepatocellular and gastric cancers (21,41-43). Hara-Chikuma and Verkman (33) demonstrated that AQP3 contributes to breast cancer cell migration by facilitating the transport of hydrogen peroxide. Another study showed that lentiviral shRNA-mediated knockdown of AQP3 in MDA-MB-231 and Bcap-37 breast cancer cell lines significantly reduced fibroblast growth factor (FGF)-2-induced cell migration (28). In addition, FGF receptor (FGFR) kinase, PI3K and mitogen-activated protein kinase kinase $1 / 2$ inhibitors reduced FGF-2-dependent increases in AQP3 expression and cell migration, indicating the involvement of the FGFR-PI3K and FGFR-ERK signaling pathways. Another study reported that siRNA-mediated knockdown of AQP3 in the $\mathrm{ER}^{+}$breast cancer cell lines T47D and MCF7 led to a prominent reduction in cell migration; upregulation of this protein in T47D cells markedly increased both processes (26). It was found that AQP3 regulates estradiol-induced breast cancer cell migration and invasion by inducing reorganization of the actin cytoskeleton (including formation of filopodia) and affecting the expression of EMT inducer molecules, favoring the metastasis of breast cancer (33). Similarly, transfection of AQP3 shRNA into MDA-MB-231 breast cancer cells reduced cell proliferation, migration and invasion (44). However, downregulation of AQP3 was also associated with a marked decrease in the viability of cells exposed to $100 \mu \mathrm{M}$ 5-fluorouracil, a fluoropyrimidine pro-drug administered to patients with breast or colorectal tumors (44).

It should be considered that the process of cancer cells attaining motility and the capacity for invasion is a complex process involving numerous molecular participants, and evaluating only one of these molecules at a time ignores the contributory effect of others; this has been demonstrated in our previous studies by the wide range of agents that inhibit blebbing $(13,14,36)$. Interpretation of data should take this into consideration. A shortcoming of siRNA knockdown is the incomplete silencing of the gene of interest, which may be more effectively achieved using gene editing techniques such as CRISPR. Further direct evidence is also required to directly connect the AQPs with the osmotic effects on blebbing.

In conclusion, the expression of AQPs 1, 3, 4 and 5 is modulated in breast cancer; all of them were upregulated in breast cancer cells compared with normal breast cells, except for AQP4, which was more intensely expressed in normal breast cells. AQP3 serves a significant role in endocrine-resistant breast cancer cell motility and invasion, and may participate in the processes leading to the blebbing of endocrine-resistant cells. Therefore, AQP3 (and potentially other AQPs) may represent additional therapeutic targets in the prevention of metastatic spread of breast cancers. 


\section{Acknowledgements}

Not applicable.

\section{Funding}

This work was supported by Kuwait University College of Graduate Studies and the Research Sector (grant no. YP02/17), a Distinguished Scholarship from Kuwait University, and a Kuwait University grant (grant no. SRUL02/13) that funds the Research Unit for Genomics, Proteomics and Cellomics Studies facilities used during the study.

\section{Availability of data and materials}

The datasets used and/or analyzed during the current study are available from the corresponding author on reasonable request.

\section{Authors' contributions}

MAK and YAL conceived the study and designed the experiments. AEA with some assistance from SK performed the experiments. AEA, MAK and YAL analyzed the data. YAL and MAK contributed reagents, materials and analytical tools. MAK, AEA and YAL drafted the manuscript. All authors read and approved the final manuscript.

\section{Ethics approval and consent to participate}

Not applicable.

\section{Patient consent for publication}

Not applicable.

\section{Competing interests}

The authors declare that they have no competing interests.

\section{References}

1. Ferlay J, Soerjomataram I, Dikshit R, Eser S, Mathers C, Rebelo M, Parkin DM, Forman D and Bray F: Cancer incidence and mortality worldwide: Sources, methods and major patterns in GLOBOCAN 2012. Int J Cancer 136: E359-E386, 2015.

2. Servick K: Breast cancer. Breast cancer: A world of differences. Science 343: 1452-1453, 2014.

3. Carey LA, Perou CM, Livasy CA, Dressler LG, Cowan D, Conway K, Karaca G, Troester MA, Tse CK, Edmiston S, et al: Race, breast cancer subtypes, and survival in the Carolina Breast Cancer Study. JAMA 295: 2492-2502, 2006.

4. Higgins MJ and Baselga J: Targeted therapies for breast cancer. J Clin Invest 121: 3797-3803, 2011.

5. Perou CM, Sørlie T, Eisen MB, van de Rijn M, Jeffrey SS, Rees CA, Pollack JR, Ross DT, Johnsen H, Akslen LA, et al: Molecular portraits of human breast tumours. Nature 406 : 747-752, 2000.

6. Sørlie T, Perou CM, Tibshirani R, Aas T, Geisler S, Johnsen H, Hastie T, Eisen MB, van de Rijn M, Jeffrey SS, et al: Gene expression patterns of breast carcinomas distinguish tumor subclasses with clinical implications. Proc Natl Acad Sci USA 98: 10869-10874, 2001

7. Wang Y and Zhou BP: Epithelial-mesenchymal Transition - A Hallmark of Breast Cancer Metastasis. Cancer Hallm 1: 38-49, 2013.

8. Al Saleh S, Al Mulla F and Luqmani YA: Estrogen receptor silencing induces epithelial to mesenchymal transition in human breast cancer cells. PLoS One 6: e20610, 2011.
9. Lamouille S, Xu J and Derynck R: Molecular mechanisms of epithelial-mesenchymal transition. Nat Rev Mol Cell Biol 15: 178-196, 2014.

10. Al Saleh S, Sharaf LH and Luqmani YA: Signalling pathways involved in endocrine resistance in breast cancer and associations with epithelial to mesenchymal transition (Review). Int J Oncol 38: 1197-1217, 2011.

11. Luqmani YA, Al Azmi A, Al Bader M, Abraham G and El Zawahri M: Modification of gene expression induced by siRNA targeting of estrogen receptor alpha in MCF7 human breast cancer cells. Int J Oncol 34: 231-242, 2009.

12. Khajah MA, Al Saleh S, Mathew PM and Luqmani YA: Differential effect of growth factors on invasion and proliferation of endocrine resistant breast cancer cells. PLoS One 7: e41847, 2012.

13. Khajah MA, Almohri I, Mathew PM and Luqmani YA: Extracellular alkaline $\mathrm{pH}$ leads to increased metastatic potential of estrogen receptor silenced endocrine resistant breast cancer cells. PLoS One 8: e76327, 2013.

14. Khajah MA, Mathew PM, Alam-Eldin NS and Luqmani YA: Bleb formation is induced by alkaline but not acidic $\mathrm{pH}$ in estrogen receptor silenced breast cancer cells. Int J Oncol 46: 1685-1698, 2015

15. Khajah MA and Luqmani YA: Involvement of Membrane Blebbing in Immunological Disorders and Cancer. Medical principles and practice: International journal of the Kuwait University. Health Sci Cent 25 (Suppl 2): 18-27, 2016.

16. Shi Z, Zhang T, Luo L, Zhao H, Cheng J, Xiang J and Zhao C: Aquaporins in human breast cancer: Identification and involvement in carcinogenesis of breast cancer. J Surg Oncol 106: 267-272, 2012.

17. Kasa P, Farran B, Prasad GLV and Nagaraju GP: Aquaporins in female specific cancers. Gene 700: 60-64, 2019.

18. López-Campos JL, Sánchez Silva R, Gómez Izquierdo L, Márquez E, Ortega Ruiz F, Cejudo P, Barrot Cortés E, Toledo Aral JJ and Echevarría M: Overexpression of Aquaporin-1 in lung adenocarcinomas and pleural mesotheliomas. Histol Histopathol 26: 451-459, 2011.

19. Yoshida T, Hojo S, Sekine S, Sawada S, Okumura T, Nagata T, Shimada Y and Tsukada K: Expression of aquaporin-1 is a poor prognostic factor for stage II and III colon cancer. Mol Clin Oncol 1: 953-958, 2013.

20. El Hindy N, Bankfalvi A, Herring A, Adamzik M, Lambertz N, Zhu Y, Siffert W, Sure U and Sandalcioglu IE: Correlation of aquaporin-1 water channel protein expression with tumor angiogenesis in human astrocytoma. Anticancer Res 33: 609-613, 2013.

21. Guo X, Sun T, Yang M, Li Z, Li Z and Gao Y: Prognostic value of combined aquaporin 3 and aquaporin 5 overexpression in hepatocellular carcinoma. BioMed Res Int 2013: 206525, 2013.

22. Otterbach F, Callies R, Adamzik M, Kimmig R, Siffert W, Schmid KW and Bankfalvi A: Aquaporin 1 (AQP1) expression is a novel characteristic feature of a particularly aggressive subgroup of basal-like breast carcinomas. Breast Cancer Res Treat 120: 67-76, 2010.

23. Qin F, Zhang H, Shao Y, Liu X, Yang L, Huang Y, Fu L, Gu F and Ma Y: Expression of aquaporin1, a water channel protein, in cytoplasm is negatively correlated with prognosis of breast cancer patients. Oncotarget 7: 8143-8154, 2016.

24. Esteva-Font C, Jin BJ and Verkman AS: Aquaporin-1 gene deletion reduces breast tumor growth and lung metastasis in tumor-producing MMTV-PyVT mice. FASEB J 28: 1446-1453, 2014.

25. Kang S, Chae YS, Lee SJ, Kang BW, Kim JG, Kim WW, Jung JH, Park HY, Jeong JH, Jeong JY, et al: Aquaporin 3 Expression Predicts Survival in Patients with HER2-positive Early Breast Cancer. Anticancer Res 35: 2775-2782, 2015.

26. Huang YT, Zhou J, Shi S, Xu HY, Qu F, Zhang D, Chen YD, Yang J, Huang HF and Sheng JZ: Identification of Estrogen Response Element in Aquaporin-3 Gene that Mediates Estrogen-induced Cell Migration and Invasion in Estrogen Receptor-positive Breast Cancer. Sci Rep 5: 12484, 2015.

27. Cao C, Sun Y, Healey S, Bi Z, Hu G, Wan S, Kouttab N, Chu W and Wan Y: EGFR-mediated expression of aquaporin-3 is involved in human skin fibroblast migration. Biochem $\mathrm{J} 400$ : 225-234, 2006

28. Cao XC, Zhang WR, Cao WF, Liu BW, Zhang F, Zhao HM, Meng R, Zhang L, Niu RF, Hao XS, et al: Aquaporin3 is required for FGF-2-induced migration of human breast cancers. PLoS One 8: e56735, 2013 . 
29. Li YB, Sun SR and Han XH: Down-regulation of AQP4 Inhibits Proliferation, Migration and Invasion of Human Breast Cancer Cells. Folia Biol (Praha) 62: 131-137, 2016.

30. Lee SJ, Chae YS, Kim JG, Kim WW, Jung JH, Park HY, Jeong JY, Park JY, Jung HJ and Kwon TH: AQP5 expression predicts survival in patients with early breast cancer. Ann Surg Oncol 21: 375-383, 2014.

31. Jung HJ, Park JY, Jeon HS and Kwon TH: Aquaporin-5: A marker protein for proliferation and migration of human breast cancer cells. PLoS One 6: e28492, 2011.

32. Livak KJ and Schmittgen TD: Analysis of relative gene expression data using real-time quantitative PCR and the 2(-Delta Delta C(T)) Method. Methods 25: 402-408, 2001.

33. Hara-Chikuma M and Verkman AS: Aquaporin-3 facilitates epidermal cell migration and proliferation during wound healing. J Mol Med (Berl) 86: 221-231, 2008.

34. Luo L, Yang R, Zhao S, Chen Y, Hong S, Wang K, Wang T, Cheng J, Zhang T and Chen D: Decreased miR-320 expression is associated with breast cancer progression, cell migration, and invasiveness via targeting Aquaporin 1. Acta Biochim Biophys Sin (Shanghai) 50: 473-480, 2018

35. Zhu Z, Jiao L, Li T, Wang H, Wei W and Qian H: Expression of AQP3 and AQP5 as a prognostic marker in triple-negative breast cancer. Oncol Lett 16: 2661-2667, 2018.

36. Khajah MA and Luqmani YA: Involvement of Membrane Blebbing in Immunological Disorders and Cancer. Med Princ Pract 25 (Suppl 2): 18-27, 2016.

37. Monzani E, Bazzotti R, Perego $\mathrm{C}$ and La Porta CA: AQP1 is not only a water channel: It contributes to cell migration through Lin7/beta-catenin. PLoS One 4: e6167, 2009.
38. Schwab A: Function and spatial distribution of ion channels and transporters in cell migration. Am J Physiol Renal Physiol 280: F739-F747, 2001.

39. Hu J and Verkman AS: Increased migration and metastatic potential of tumor cells expressing aquaporin water channels. FASEB J 20: 1892-1894, 2006.

40. Khajah MA, Mathew PM and Luqmani YA: $\mathrm{Na}^{+} / \mathrm{K}^{+}$ATPase activity promotes invasion of endocrine resistant breast cancer cells. PLoS One 13: e0193779, 2018.

41. Chen J, Wang T, Zhou YC, Gao F, Zhang ZH, Xu H, Wang SL and Shen LZ: Aquaporin 3 promotes epithelial-mesenchymal transition in gastric cancer. J Exp Clin Cancer Res 33: 38, 2014.

42. Li A, Lu D, Zhang Y, Li J, Fang Y, Li F and Sun J: Critical role of aquaporin-3 in epidermal growth factor-induced migration of colorectal carcinoma cells and its clinical significance. Oncol Rep 29: 535-540, 2013.

43. Liu S, Zhang S, Jiang H, Yang Y and Jiang Y: Co-expression of AQP3 and AQP5 in esophageal squamous cell carcinoma correlates with aggressive tumor progression and poor prognosis. Med Oncol 30: 636, 2013.

44. Arif M, Kitchen P, Conner MT, Hill EJ, Nagel D, Bill RM, Dunmore SJ, Armesilla AL, Gross S, Carmichael AR, et al: Downregulation of aquaporin 3 inhibits cellular proliferation, migration and invasion in the MDA-MB-231 breast cancer cell line. Oncol Lett 16: 713-720, 2018 